Enfermagem Brasil 2018;17(4):318-25

\title{
ARTIGO ORIGINAL \\ (Des) conhecimento sobre a prática da violência obstétrica
}

Nirliane Ribeiro Barbosa, M.Sc.*, Thamyres Queiroz de Lima**, Luciana de Amorim Barros, M.Sc. ${ }^{* *}$, Cleide Ferreira de Amorim Cotta ${ }^{* * *}$

${ }^{*}$ Enfermeira pela Universidade Federal de Alagoas (UFAL), Docente Adjunta na Universidade Federal de Alagoas, ${ }^{*}$ Enfermeira pela Universidade Federal de Alagoas (UFAL), Campus Arapiraca, Pós-Graduada em Saúde Pública, ${ }^{* \star *}$ Enfermeira pelo Centro de Estudos Superiores de Maceió, Docente Assistente na Universidade Federal de Alagoas, ${ }^{* * \star * E n f e r m e i r a ~ O b s t e t r a ~}$ do Hospital Beneficente Nossa Senhora do Bom Conselho

Recebido em 13 de dezembro de 2017; aceito em 22 de agosto de 2018.

Endereço de correspondência: Nirliane Ribeiro Barbosa, Rua Alan Kardec, 430 Santa Esmeralda 57312-220 Arapiraca AL, E-mail: nirliane@hotmail.com; Thamyres Queiroz de Lima: thamyresq@hotmail.com; Luciana de Amorim Barros: lukota_amorim@hotmail.com; Cleide Ferreira de Amorim Cotta: cleidecotta@uol.com.br

\section{Resumo}

O objetivo deste estudo foi conhecer a percepção dos profissionais de saúde sobre violência obstétrica. Trata-se de uma pesquisa qualitativa, exploratória e de caráter descritivo nas maternidades do município de Arapiraca/AL. A coleta de dados foi realizada com 30 profissionais de saúde de ensino superior (entre enfermeiros, fisioterapeutas, médicos e assistentes sociais), mediante assinatura do Termo de Consentimento Livre e Esclarecido, baseada em um roteiro de entrevista semiestruturada, adaptada de Aguiar e D'Oliveira. Para a análise dos dados, foi realizada a técnica de análise de conteúdo de Bardin. A partir da análise das falas decorrentes das entrevistas emergiu a categoria, a saber: $O$ (des) conhecimento sobre a prática da violência obstétrica. É evidente que a violência obstétrica é um termo ainda pouco conhecido entre os profissionais de saúde, no entanto muitos entrevistados puderam perceber durante sua jornada de trabalho maus tratos contra a parturiente. Diante disto é preciso divulgar a temática violência obstétrica entre profissionais de saúde, realizando educação continuada dentro dos serviços de saúde, além de incorporar na legislação brasileira as medidas cabíveis para tais atos.

Palavras-chave: violência contra a mulher, obstetrícia, pessoal de saúde, Enfermagem.

\section{Abstract \\ (Dis)knowledge on the practice of obstetric violence}

The objective of this study was to know the perception of health professionals on obstetric violence. This is a qualitative, exploratory and descriptive study in the maternities of the city of Arapiraca/AL, Brazil. Data collection was performed with 30 higher education health professionals (among nurses, physical therapists, physicians and social workers), by signing the Informed Consent Term, based on a semi-structured interview script, adapted from Aguiar and D'Oliveira. For the data analysis, the Bardin content analysis technique was performed. From the analysis of the speeches resulting from the interviews, the category emerged, namely: The (dis) knowledge about the practice of obstetric violence. It is evident that obstetric violence is a term still little known among health professionals, however many interviewees were able to perceive during their working day mistreatment against the parturient. Faced with this, it is necessary to publicize the issue of obstetric violence among health professionals, carrying out continuing education within the health services, and incorporate into Brazilian legislation the appropriate measures for such acts.

Key-words: violence against women, obstetrics, health personnel, Nursing.

\section{Resumen}

\section{(Des)conocimiento sobre la práctica de la violencia obstétrica}

El objetivo de este estudio fue conocer la percepción de los profesionales de salud sobre violencia obstétrica. Se trata de una investigación cualitativa, exploratoria y de carácter descriptivo en las maternidades del municipio de Arapiraca/AL. La recolección de datos fue 
realizada con 30 profesionales de salud de enseñanza superior (entre enfermeros, fisioterapeutas, médicos y asistentes sociales), mediante la firma del Término de Consentimiento Libre y Esclarecido, basada en un guión de entrevista semiestructurada, adaptada de Aguiar y De Oliveira. Para el análisis de los datos, se realizó la técnica de análisis de contenido de Bardin. A partir del análisis de los discursos resultantes de las entrevistas surgió la categoría, a saber: $\mathrm{O}$ (des) conocimiento sobre la práctica de la violencia obstétrica. Es evidente que la violencia obstétrica es un término aún poco conocido entre los profesionales de la salud, pero muchos entrevistados pudieron percibir durante su jornada de trabajo malos tratos contra la parturienta. Ante esto es necesario divulgar la temática violencia obstétrica entre profesionales de salud, realizando una educación continuada dentro de los servicios de salud, además de incorporar en la legislación brasileña las medidas apropiadas para tales actos.

Palabras-clave: violencia contra la mujer, obstetricia, personal de salud, Enfermería.

Introdução

Na década de 80, com a criação do Sistema Único de Saúde (SUS) no Brasil, emergiu a expressão humanização na área da saúde e posteriormente com a implementação do Programa de Humanização no Pré-natal e Nascimento em 2002, pelo Ministério da Saúde, passa a ser exigida na assistência do pré-natal, parto e puerpério. Para efetivação deste princípio de humanização é essencial o acolhimento da mulher e do recém-nascido, enfocando a adoção de valores de autonomia e protagonismo, estimulando a corresponsabilidade entre os agentes atuantes no parto [1].

Em seguida, nesta mesma perspectiva de assistência humanizada na gravidez, parto e puerpério, foi implementada a estratégia Rede Cegonha, definida pela Portaria № 1.459 em 24 de junho de 2011, visando à sistematização e institucionalização de uma rede de cuidados estabelecendo metas relacionadas às ações de atenção à saúde, dentre estas se encontram as taxas de: acompanhante, episiotomia, parto normal em posição verticalizada, contato imediato pele a pele efetivo, cesariana, e de parturientes que receberam método não farmacológico para alívio de dor [2]. A apresentação destes indicadores nas maternidades vinculadas a estratégia Rede Cegonha no município de Arapiraca/AL revela uma melhora da assistência a parturiente ao longo do ano de 2014; contudo, os indicadores sobre taxa de cesariana foram aquém das metas preconizadas para estas maternidades [3]. A partir desta realidade infere-se que a assistência à mulher no exercício de sua saúde sexual e reprodutiva está inadequada.

Embora o tema seja considerado "recente", o sofrimento das mulheres com a assistência ao parto é registrado em diferentes momentos históricos, ainda que sob denominações diversas, encontrando respostas em distintos contextos, e comumente tendo um impacto importante na mudança das práticas de cuidado no ciclo gravídico-puerperal [4].

violência nas maternidades, cometidas por profissionais de saúde, especialmente da categoria enfermagem. $O$ enfermeiro é habilitado a realizar assistência à gestante, parturiente e puérpera, acompanhando a evolução do trabalho de parto e execução do parto sem distócia, segundo a Lei do Exercício Profissional da Enfermagem (Lei $N^{\circ}$ 7.498/86) [8]. Segundo pesquisa realizada, em 2010, pela Fundação Perseu Abramo: "Mulheres brasileiras e Gênero nos espaços público e privado", uma em cada quatro mulheres brasileiras sofre violência no parto [9].

No Brasil há lacunas sobre legislação específica para violência obstétrica. Em contrapartida, a Assembléia Nacional da República Bolivariana da Venezuela aprovou em 2007 a Lei Orgânica sobre o Direito das Mulheres a uma Vida Livre da Violência, que tipifica, entre as 19 formas de violência contra a mulher, a violência obstétrica. Esta pode ser definida como:

\footnotetext{
"a apropriação do corpo e processos reprodutivos das mulheres por profissional de saúde, que se expressa em um tratamento desumanizador, em um abuso de medicalização e patologização dos processos naturais, trazendo consigo perda de autonomia e capacidade de decidir livremente sobre seus corpos e sexualidade, impactando negativamente na qualidade de vida das mulheres [10]."
}

Considerando-se, ainda, que frequentemente as mulheres escolhem ter o parto cesáreo por medo das atitudes dos profissionais durante a assistência ao trabalho de parto, por já ter presenciado ou ouvido de alguém relatos de violência. Isso nos faz refletir por que ainda acontece este tipo de violência nas maternidades, será por desconhecimento dos profissionais 
sobre a legislação? Por não perceberem a intensidade de palavras e atitudes dirigidas às usuárias? Ou a sobrecarga de trabalho? Diante disso tem-se como objetivo da pesquisa conhecer a percepção dos profissionais de saúde sobre violência obstétrica.

\section{Material e métodos}

Trata-se de uma pesquisa qualitativa, exploratória e de caráter descritivo, realizado em duas maternidades vinculadas a estratégia Rede Cegonha do Município de Arapiraca/AL. Foram convidados 43 profissionais de saúde de ensino superior, destes 30 aceitaram participar (quatorze enfermeiros, oito fisioterapeutas, cinco médicos e três assistentes sociais). Foram incluídos na pesquisa todos os profissionais de saúde de ensino superior que apresentaram vínculo empregatício nas maternidades estudadas no período da entrevista; encontraram-se no momento da pesquisa atuando na assistência ao parto e/ou fazendo parte do processo de acolhimento das mulheres atendidas nas maternidades. Foram critérios de exclusão da pesquisa todos os profissionais que não estiveram presentes na instituição no período da pesquisa devido licença ou afastamento; não se disponibilizaram participar da pesquisa.

O projeto de pesquisa foi autorizado pelos locais do estudo, bem como aprovado pelo Comitê de Ética em Pesquisa da Universidade Federal de Alagoas, segundo o parecer $\mathrm{N}^{\circ}$ 1.350.370. A aceitação dos sujeitos para participação do estudo foi mediante assinatura do Termo de Consentimento Livre e Esclarecido. A coleta de dados foi realizada no período de dezembro de 2015 a fevereiro de 2016, nos próprios locais de estudo, de segunda à sexta-feira, no período da tarde, entre um atendimento e outro, em momentos de descanso ou de menor demanda, mediante roteiro de entrevista semiestruturada, adaptada de entrevista realizada no estudo de Aguiar e D'Oliveira [5]. Este roteiro continha 10 questões discursivas que indagavam sobre: idade, profissão, tempo de trabalho, número de instituições que trabalha, número de filhos, tipo de parto(s), conhecimento sobre termo violência obstétrica e quem pode cometê-la, presença de algum tipo de maus tratos contra a mulher durante sua jornada de trabalho, conhecimento de alguma lei que protege a mulher contra qualquer tipo de violência, conhecimento sobre quais são os meios de denunciar a violência obstétrica.

Houve uma grande dificuldade na captação dos profissionais pela demanda das maternidades, acarretando a justificativa de algumas recusas. Todas as entrevistas foram gravadas, guardadas em arquivo digital, para posterior transcrição e análise dos dados. Para a análise dos dados, foi utilizada a técnica de análise de conteúdo de Bardin. Este método utiliza três fases básicas: pré-análise, exploração do material e o tratamento dos resultados obtidos e interpretação [12]. Os profissionais de saúde foram identificados por um número, de 1 a 30 , que correspondeu à ordem das entrevistas, para assegurar a identidade dos mesmos.

Resultados

Foram convidados 43 profissionais de saúde de nível superior em ambos os locais de estudo para participar da pesquisa, 30 aceitaram, sendo quatorze enfermeiros, oito fisioterapeutas, cinco médicos e três assistentes sociais. Quanto ao sexo houve uma prevalência do feminino, vinte e quatro $(80 \%)$, e seis $(20 \%)$ do sexo masculino (um enfermeiro e cinco médicos). Entre essas, 17 possuem filhos. Quanto a faixa etária a maioria possui de 31 a 40 anos de idade, sendo a menor idade encontrada foi 24 anos e a maior 66 anos. Quando questionados sobre o número de instituições em que trabalham, a maioria trabalha em uma, contudo um profissional trabalha em seis instituições. Os dados mostram que os profissionais, no geral, são relativamente novos na instituição estudada, pois a maior parte trabalha de um a cinco anos, contudo já possuem certa experiência na área de atuação, obtendo-se uma média de 10,3 anos de conclusão da graduação.

A partir da análise das falas decorrentes das entrevistas emergiu a categoria: $\mathrm{O}$ (des) conhecimento sobre a prática da violência obstétrica.

\section{O (des)conhecimento sobre a prática da violência obstétrica}

A maioria dos entrevistados respondeu que conheciam o termo violência obstétrica $(90 \%)$, apesar de a Fisioterapia ser a categoria profissional que mais teve dificuldade para explicar este conceito. Alguns referiram que não conheciam porque eles não estão ligados diretamente ao parto, e entendem que eles não têm a "obrigação" de saber. Contudo, quando 
começavam a definir, constatou-se que alguns não conheciam a dimensão desta prática. Associam mais ao parto normal ou somente ao uso de métodos invasivos no momento do parto.

[...] É... esses tipos de parto, um parto que... muito invasivo né?! Que invés de fazerem uma cesárea eles optam por fazerem essa violência, né?! Que é parto fórceps, é... episiotomia, né?! isso é violência obstétrica. (Profissional 3)

Dentre os entrevistados apenas uma enfermeira citou que a violência obstétrica compreende tanto o período gestacional, quanto trabalho de parto, parto e aborto.

Conheço. Assim, é... violência obstétrica é uma, uma violência que ela é acometida a mulher nesse período né?! de gestação, no período de trabalho de parto, no período de parto, até no período da gestação né?! ou é... um abortamento ou outra condição que seja relacionada [...] (Profissional 10)

Quase metade dos profissionais entrevistados (14) referiu ser a violência obstétrica praticada por profissionais ligados diretamente ao parto, como técnico de enfermagem, enfermeiro, médico ou parteira.

O médico obstetra, a enfermeira obstetra, a parteira. (Profissional 8)

Neste estudo foi levantado pelos entrevistados (seis profissionais (20\%)) que o próprio acompanhante pode vir a causar algum ato violento contra a mulher neste período.

[...] acho que até mesmo o acompanhante, sei lá, a meu ver, pode tá cometendo. Qualquer pessoa que esteja acompanhando a gestante naquele momento, acho que até família [...] (Profissional 2)

E dezesseis participantes do estudo (53,33\%) afirmaram que qualquer profissional de saúde pode estar envolvido em violência obstétrica, incluindo desde a recepção, pessoal da limpeza até da copa.

"Qualquer profissional da área, independente se é obstetra ou não. Às vezes nós temos agressões por pediatras neonatologistas, ou parteiras, não tem uma, uma classe que seja definida, pra cometer essa violência. Ass vezes comete violência até da... da portaria né?! até da recepção a mulher as vezes já começa a sofrer violência." (Profissional 23)

"[...] Desde o pessoal da limpeza, quanto pessoal da burocracia, de recepção, de copa, quanto os profissionais da saúde [...]." (Profissional 27)

Três profissionais (10\%) referiram que praticar este tipo de violência depende de pessoa para pessoa, da essência de cada um, e que é influenciado pela educação/formação que obteve.

[...] porque muitas vezes depende de cada, da educação que você teve, acho que tem fatores externos em relação a isso. Que tipo de treinamento você teve, como era a abordagem, né?! como foi a abordagem de uma gestante ou como era essa abordagem anteriormente. Porque você vai muito do que, da forma como você aprende. Muitas vezes você aprende já errado achando que aquela é a forma certa de lidar, acho que a gente vê muito isso no dia-a-dia [...] (Profissional 25)

Quando questionados sobre já ter presenciado algum tipo de maus tratos contra a mulher durante a jornada de trabalho, quinze $(50 \%)$ afirmaram que já presenciaram algum tipo de violência obstétrica durante sua jornada de trabalho, seja psicológica, física ou práticas obstétricas como toques vaginais frequentes, manobra de Kristeller, uso rotineiro da posição litotômica durante o trabalho de parto e de episiotomia.

"[...] Já vi é... paciente que foi estuprada ser praticamente obrigada a ter parto normal sem querer, ser tocada sem querer [...]." (Profissional 23)

"[...] De impulsionar ela fazer alguma coisa que ela não tem vontade naquele momento, é... obrigar ela a fazer algumas posições que ela também não se sente à vontade, obrigar a fazer um toque vaginal que ela naquele momento não quer, então tudo isso é uma forma de violência 
obstétrica. Como também aquela questão de uma episiotomia que às vezes nem precisa e que ela nem tem consciência que precisa ou não, mas o profissional vai fazer de qualquer forma, sem necessidade. É... um kristeller, que hoje não é indicado e alguns profissionais ainda, ainda bem que são bem menos, mas ainda hoje acaba fazendo [...]." (Profissional 27)

Discussão

Os dados desta pesquisa relativos à caracterização dos sujeitos corroboram outros estudos [12-15]. Entretanto, no presente estudo houve uma predominância do profissional enfermeiro $(46,6 \%)$, diferente de outros nos quais os médicos foram profissionais mais entrevistados. Em Santos [14], participaram 13 profissionais de saúde, dos quais cinco enfermeiros obstetras e oito médicos obstetras, enquanto em Oliveira [15], um total de dez enfermeiros obstetras e 14 médicos obstetras. Ambos estudos não deixaram claro se apresentam um número maior de médicos do que as outras categorias ou se obteve uma maior adesão por parte destes para realização das pesquisas.

Outro aspecto importante é que a sobrecarga de trabalho não foi uma fala emergente nas entrevistas e a maioria dos participantes não possuía vínculo empregatício com outras instituições, no entanto pode acontecer sobrecarga diante da demanda das instituições em estudo e carga horária neste serviço. Tal excesso pode ocasionar, conforme apontam Aguiar, D'Oliveira e Schraiber [13], um esgotamento físico e emocional do profissional, assim como a dificuldade de refletir sobre sua prática, resultante de um ritmo de trabalho alienante associado à precariedade de recursos.

Ao abordar sobre o que os profissionais entendem sobre o termo violência obstétrica, a maioria afirmou que o conhecia, semelhante resultado foi encontrado na pesquisa de Faneite et al. [12], com um percentual de $89,2 \%$. No entanto, isso não significa que realmente sabem distingui-lo, o que foi notório na fala de uma enfermeira que confundiu com violência doméstica, e ainda ao referirem ser praticado apenas por profissionais ligados diretamente ao parto, como técnico de enfermagem, enfermeiro, médico ou parteira. Leal [16] em seu estudo pode constatar que as enfermeiras obstétricas percebem a existência de violência obstétrica de forma limitada, não reconhecendo as intervenções como uma prática violenta. Além disso, quando há o reconhecimento de tais procedimentos, existe a justificativa da ajuda à parturiente para a realização de tais condutas. Ficando evidente que os participantes associam mais frequentemente o termo violência a algo que consideram de maior gravidade, como a violência física e sexual [14]. Isso mostra que nem todos os profissionais buscam se atualizar e quando se fala em violência contra a mulher habitualmente relaciona-se a forma sexual e doméstica [13].

Segundo o Dossiê Violência Obstétrica [10], em seu Artigo 51, os seguintes atos são considerados típicos de violência obstétrica: não atender oportuna e eficazmente as emergências obstétricas; obrigar a mulher a parir em posição supina, existindo os meios necessários para a realização do parto vertical; obstaculizar o contato precoce do bebê com sua mãe, sem causa médica justificada; alterar o processo natural do parto de baixo risco, mediante o uso de técnicas de aceleração, ou praticar o parto cesariano, existindo condições para o parto natural, sem obter o consentimento voluntário, expresso e informado da mulher.

Muitos casos de manobra de kristeller (esforço de puxo prolongado e dirigido durante o segundo estágio do trabalho de parto), uso de rotina da posição supina durante o trabalho de parto, exames vaginais frequentes e o uso rotineiro de episiotomia foram presenciados pelos entrevistados durante a jornada de trabalho, práticas obstétricas que são prejudiciais, se usadas de modo inadequado, segundo as recomendadas da Organização Mundial da Saúde (OMS) desde 1996 [17] e reafirmadas pela Diretriz Nacional de Assistência ao Parto Normal [18], documentos que orientam as boas práticas obstétricas. Em consonância com este achado, na pesquisa de Cardoso [19], 70\% julgaram que não cometeram violência obstétrica, todavia, quando questionados em relação à perspectiva e à percepção que tinham acerca do colega de trabalho, $80 \%$ dos entrevistados referiram já ter presenciado colegas cometendo algum tipo de violência obstétrica. Essa discussão mostra que os (as) profissionais das maternidades devem ser estimulados (as) com mais intensidade para as boas práticas obstétricas.

Andrade et al. [20] também puderam evidenciar em um hospital escola de alta complexidade e de referência do Ministério da Saúde para a assistência materno-infantil em Recife, Pernambuco, em que aproximadamente $87 \%$ das pacientes sofreram algum tipo de violência durante 0 trabalho de parto e parto, considerando 0 uso de intervenções desnecessárias. 
Os profissionais foram pertinentes ao afirmarem que qualquer profissional de saúde pode cometer violência obstétrica, envolvendo desde a recepção, pessoal da limpeza, da copa até os profissionais coadjuvantes do parto, uma vez que, segundo o Dossiê da Violência Obstétrica [10], esse tipo de violência pode ser exercido por todos os trabalhadores dos serviços público ou privado que atuem nos centros de saúde, tanto profissionais (médicos/as, trabalhadores/ as sociais, psicólogos/as) como contribuintes (mucamas/o, enfermeiros, pessoal administrativo, entre outros). Desfecho diferente foi encontrado por Faneite, Feo e Merlo [12], que, ao interrogar profissionais da área obstétrica sobre por quem poderia ser exercida a violência obstétrica, $82,4 \%$ responderam que poderia ser exercida por qualquer profissional de saúde e $17,6 \%$ consideraram que a mesma era exercida somente pelo médico obstetra.

Na pesquisa de Pereira, Domínguez e Merlo [21], 26,3\% das pacientes entrevistadas manifestaram ter sofrido maus-tratos, desrespeito, atitude de desprezo ou ridículo, agressão verbal ou física, durante sua atenção por parte do profissional de saúde, e que o médico obstetra foi relatado pelas pacientes como um dos principais agressores. Este fato foi confirmado por Andrade e seus coautores [20], pois observaram significante associação entre a violência obstétrica e mulheres assistidas por profissional médico.

Em consequência a esta realidade, as mulheres sentem-se incapazes, impotentes e reduzidas a objetos, no contexto em que deveriam se tornar personagem principal. Os efeitos nocivos que podem ser acarretados com um atendimento inadequado são vários e podem gerar danos a curto prazo como: insatisfações com o serviço, intercorrências puerperais, traumas psicológicos graves ou até mesmo óbito materno e/ou neonatal; e a longo prazo, como: o impacto negativo na qualidade de vida das mulheres, problemas conjugais e na sexualidade da mulher $[22,23]$.

Destarte é preciso divulgar a temática violência obstétrica, tanto entre gestantes, durante o pré-natal, enfatizando seus direitos na assistência a gestação, parto e puerpério e contribuindo para empoderá-las, quando entre profissionais de saúde, de nível superior ou não. É necessário, pois, investir desde a graduação dos (as) profissionais de saúde, buscando incentivar o cuidado humanizado, em qualquer que seja o tipo de parto, o uso de boas práticas obstétricas e promovendo que o parto fisiológico aconteça. Não obstante, como mencionado por Barbosa [24], ainda há o predomínio da formação pautada no tecnicismo e no uso indiscriminado das tecnologias e intervenções iatrogênicas na assistência obstétrica de baixo risco. Além disso, os profissionais devem buscar atualizações sempre através de eventos científicos, especializações e em pesquisas na área, principalmente os profissionais da enfermagem e medicina, mencionados como principais responsáveis por cometer atos de desrespeito contra a mulher no período gravídico-puerperal neste trabalho.

Assim, permanece evidente a necessidade de punição dos responsáveis por atos característicos de violência obstétrica, e para isso os órgãos competentes devem ter conhecimento deste tipo de crime, acatar tais denúncias e incorporar na legislação brasileira as medidas cabíveis. As denúncias são de suma importância para que o SUS e os órgãos judiciais apurem os fatos e possam, por meios destas denúncias, modificar a realidade brasileira, garantindo assim uma assistência obstétrica equânime, resolutiva e humana [25]. Para isso, Sadler [26] em sua pesquisa sugere a implementação de sistemas de notificação que permitam as mulheres e os profissionais denunciar casos de violência obstétrica. Um próximo passo, na perspectiva de Bohren et al. [27], seria o desenvolvimento de ferramentas validadas e confiáveis para medir os maus-tratos de mulheres durante o parto, bem como intervenções para preveni-los e promover cuidados respeitosos.

É necessário compreender que não são necessárias grandes mudanças estruturais e nem um investimento em altas tecnologias e sim um olhar mais atencioso do (a) profissional, reconhecendo as individualidades da paciente, buscando oferecer uma assistência baseada em evidências científicas. Além disso, suas ações devem ser fundamentadas nas diretrizes da bioética e do Código de Ética de cada profissão e, portanto, nos seguintes princípios: autonomia, beneficência, não maleficência e justiça [14].

Nota-se uma escassez de material sobre o assunto na literatura brasileira, o que dificultou o diálogo com outras pesquisas e públicos semelhantes neste estudo. Outro ponto limitante deste estudo foi não ter realizado a entrevista entre profissionais de saúde de nível médio, e a mesma ter ocorrido nos próprios locais de estudo, o que pode ter constrangido os entrevistados a falar sobre os companheiros de trabalho e sobre a instituição. 
O estudo possibilitou alcançar o objetivo de analisar a percepção dos profissionais de saúde sobre violência obstétrica. De acordo com o que foi exposto, é notório que a violência obstétrica é um termo ainda pouco conhecido entre os profissionais de saúde na realidade estudada, embora estes sejam atores responsáveis por proporcionar um atendimento qualificado nesse período gravídico-puerperal. Para além do termo, muitos não reconhecem atos típicos de violência obstétrica, nem se reconhecem capazes de cometê-los. Contudo ao trocar os papeis estes identificaram condutas inadequadas nos atendimentos, aproximando-se da prática da violência obstétrica.

Referências

1. Brasil. Ministério da Saúde. Secretaria executiva. Programa de humanização do parto: humanização no pré-natal e nascimento. Brasília: Ministério da Saúde; 2002.

2. Brasil. Ministério da Saúde. Secretaria de Atenção à Saúde. Manual Prático para Implementação da Rede Cegonha. Brasília: Ministério da Saúde; 2011.

3. Barbosa NR, Lima TQ, Lira KRB, Silva WS, Pinto EA, Santos JKC. Indicadores da Rede Cegonha nas maternidades de Arapiraca-AL. Comunicação Coordenada do 69ำ Congresso Brasileiro de Enfermagem. Maceió-AL; 2017.

4. Diniz SG, Salgado HO, Andrezzo HFA, Carvalho PGC, Carvalho PCA, Aguiar CA, Niy DY. Abuse and disrespect in childbirth care as a public health issue in brazil: origins, definitions, impacts on maternal health, and proposals for its prevention. J Hum Growth Dev 2015;25(3):377-384.

5. Silveira SC, Camargo BV, Crepaldi MA. Assistência ao parto na maternidade: representações sociais de mulheres assistidas e profissionais de saúde. Psicol Reflex Crit 2010;23(1):1-10.

6. Aguiar JM, D'Oliveira AFPL. Violência institucional em maternidades públicas: hostilidade ao invés de acolhimento como uma questão de gênero [Tese]. São Paulo: Faculdade de Medicina da Universidade de São Paulo; 2010.

7. Pulhez MMA. "Violência obstétrica" e as disputas em torno dos direitos sexuais e reprodutivos. In: Anais Eletrônicos do Seminário Internacional Fazendo Gênero, 2013; Florianópolis. [citado 2015 Jan 1]. Disponível em: http://www.fazendogenero.ufsc.br/10/resources/anais/20/1386706264_ARQUIVO_Mari anaMarquesPulhez.pdf

8. Brasil. Lei n. 7.498, de 25 de junho de 1986. Dispõe sobre a regulamentação do exercício da enfermagem, e dá outras providências. Brasília: Diário Oficial da República Federativa do Brasil; 1986.

9. Bokany V, Dias G, Alba D, Rosas W, Figueiredo N. Mulheres brasileiras e gênero nos espaços públicos e privado. Fundação Perseu Abramo e SESC; 2010 [citado 2016 maio 29]. Disponível em: http://novo.fpabramo.org.br/ sites/default/files/pesquisaintegra_0.pdf

10. Dossiê da Violência Obstétrica. Parto do princípio. Mulheres em Rede pela Maternidade Ativa. Violência Obstétrica - "Parirás com dor". Brasília: Senado Federal; 2012.

11. Leopardi MT, Rodrigues MSP. O método de análise de conteúdo: uma versão para enfermeiros. 1.ed. Fortaleza: Fundação Cearense de Pesquisa e Cultura; 1999.

12. Faneite DRASJ, Feo A, Merlo JT. Grado de conocimiento de violencia obstétrica por el personal de salud. Rev Obstet Ginecol Venez 2012;72(1):4-12.

13. Aguiar JM, D'Oliveira AFPL, Schraiber LB. Violência institucional, autoridade médica e poder nas maternidades sob a ótica dos profissionais de saúde. Cad Saúde Pública 2013;29(11):2287-96.

14. Santos MG. A violência obstétrica sob o olhar de profissionais de saúde [Dissertação]. Goiânia: Universidade Federal de Goiás, Faculdade de Enfermagem (FEN), Programa de Pós-Graduação em Enfermagem; 2017.

15. Oliveira VJ, Penna CMM. O discurso da violência obstétrica na voz das mulheres e dos profissionais de saúde. Texto Contexto Enferm 2017;26(2):e06500015.

16. Leal SYP, Lima VLA, Silva AF, Soares PDFL, Santana LR, Pereira A. Percepção de enfermeiras obstétricas acerca da violência obstétrica. Cogitare Enferm 2018;(23)2: e52473. 
17. Organização Mundial da Saúde. Tecnologia apropriada para partos e nascimentos. Recomendações da Organização Mundial de Saúde. Maternidade Segura. Assistência ao parto normal: um guia prático. Genebra: OMS; 1996.

18. Brasil. Ministério da Saúde. Diretriz nacional de assistência ao parto normal. Relatório de recomendação. Comissão Nacional de Incorporação de Tecnologia no SUS. Brasília: Ministério da Saúde; 2016.

19. Cardoso FJC, Costa ACM, Almeida MM, Santos TS, Oliveira FBM. Violência obstétrica institucional no parto: percepção de profissionais da saúde. Rev Enferm UFPE on line 2017; 11(9):3346-53.

20. Andrade PON, Silva JQP, Diniz CMM, Caminha MFC. Fatores associados à violência obstétrica na assistência ao parto vaginal em uma maternidade de alta complexidade em Recife, Pernambuco. Rev Bras Saúde Matern Infant 2016;16(1):29-37.

21. Pereira DCJ, Domínguez AL, Merlo JT. Violencia obstétrica desde la perspectiva de la paciente. Rev Obstet Ginecol Venez 2015;75(2):81-90.

22. Silva ISA, Santos MAES, Pereira MFLF, Ferraz RSR. Percepção social de puérperas sobre violência no trabalho de parto e parto: revisão integrativa [TCC]. Recife: Curso de Graduação em Enfermagem da Faculdade Integrada de Pernambuco; 2016.

23. Guedes LLB. A assistência do (a) enfermeiro(a) à parturiente no contexto hospitalar: um olhar sobre a violência obstétrica [TCC]. Governador Mangabeira: Faculdade Maria Milza; 2017.

24. Barbosa LC, Fabbro MRC, Machado GPR. Violência obstétrica: revisão integrativa de pesquisas qualitativas. Av Enferm 2017;35(2):190-207.

25. Matoso LML. O papel do enfermeiro frente à violência obstétrica. C\&D-Revista Eletrônica da FAINOR 2018;11(1):49-65.

26. Sadler M, Santos MJDS, Ruiz-Berdún D, Rojas GL, Skoko E, Gillen P, Clausen JA. Moving beyond disrespect and abuse: addressing the structural dimensions of obstetric violence, Reproductive Health Matters 2016;24:47-55. http://doi: 10.1016/j.rhm.2016.04.002.

27. Bohren MA, Vogel JP, Hunter EC, Lutsiv O, Makh SK, Souza JP, et al. (2015) The mistreatment of women during childbirth in health facilities globally: A mixed-methods systematic review. PLoS Med 12(6): e1001847. http://doi:10.1371/journal. pmed.1001847. 